\title{
THE MODULAR CLASS OF A LIE ALGEBROID COMORPHISM
}

\author{
RAQUEL CASEIRO
}

\begin{abstract}
We introduce the definition of modular class of a Lie algebroid comorphism and exploit some of its properties.
\end{abstract}

\section{Introduction}

The modular class of a Poisson manifold $M$ is an element of the first Poisson cohomology group $H_{\pi}^{1}(M)$, which measures the obstruction to the existence of a measure in $M$ invariant under all hamiltonian diffeomorphisms $([9,12])$. This notion was extended to Lie algebroids by Evan, Lu and Weinstein [3] who showed that the modular class of the cotangent bundle of a Poisson manifold is twice the modular class of the Poisson structure. Grabowski, Marmo and Michor [6] introduced the modular class of a Lie algebroid morphism and this was more deeply studied by Kosmann-Schwarzbach, Laurent-Gengoux and Weinstein in [7] and [8]. In a recent paper [2], the notion of modular class of a Poisson map was given and some of its properties studied. Even more recently Grabowski [5] generalizes all these definitions introducing the modular class of skew algebroid relations. In this paper we exploit the definition of the modular class of a Lie algebroid comorphism, following the approach in [2].

\section{The modular class of a Lie algebroid}

Let $A \rightarrow M$ be a Lie algebroid over $M$, with anchor $\rho: A \rightarrow T M$ and Lie bracket $[\cdot, \cdot]: \Gamma(A) \times \Gamma(A) \rightarrow \Gamma(A)$. We will denote by $\Omega^{k}(A) \equiv \Gamma\left(\wedge^{k} A^{*}\right)$ the $A$-forms and by $\mathfrak{X}^{k}(A) \equiv \Gamma\left(\wedge^{k} A\right)$ the $A$-multivector fields. Recall that the $A$-differential $\mathrm{d}_{A}: \Omega^{k}(A) \rightarrow \Omega^{k+1}(A)$ is given by

$$
\begin{aligned}
\mathrm{d}_{A} \alpha\left(X_{0}, X_{1} \ldots, X_{n}\right) & =\sum_{k=1}^{n}(-1)^{i} \rho\left(X_{i}\right) \ldots \alpha\left(X_{0}, \ldots, \hat{X}_{i}, \ldots, X_{n}\right) \\
& +\sum_{0 \leq i<j \leq k}(-1)^{i+j} \alpha\left(\left[X_{i}, X_{j}\right], X_{0}, \ldots, \hat{X}_{i}, \ldots \hat{X}_{j}, \ldots, X_{n}\right)
\end{aligned}
$$

and turns $\Omega^{\bullet}(A)$ into a complex whose cohomology is called the Lie algebroid cohomology and will be denoted by $H^{\bullet}(A)$.

Example 2.1. In case $A=T M$, the Lie algebroid cohomology is the De Rham cohomology.

Example 2.2. For any Poisson manifold $(M, \pi)$ there is a natural Lie algebroid structure on its cotangent bundle $T^{*} M$ : the anchor is $\rho=\pi^{\sharp}$ and the Lie bracket on sections of $A=T^{*} M$, i.e., on one forms, is given by:

$$
[\alpha, \beta]=£_{\pi^{\sharp} \alpha} \beta-£_{\pi^{\sharp} \beta} \alpha-\mathrm{d} \pi(\alpha, \beta) .
$$

The Poisson cohomology of $(M, \pi)$ is just the Lie algebroid cohomology of $T^{*} M$.

RC is partially supported by PTDC/MAT/099880/2008. 
A morphism between two Lie algebroids $A \rightarrow M$ and $B \rightarrow N$ is a vector bundle $\operatorname{map}(\Phi, \phi)$

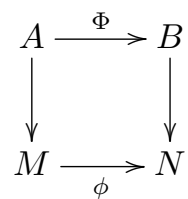

such that the dual vector bundle map $\Phi^{*}:\left(\Omega^{\bullet}(B), \mathrm{d}_{B}\right) \rightarrow\left(\Omega^{\bullet}(A), \mathrm{d}_{A}\right)$ is a chain map.

The most basic example of a Lie algebroid morphism is the tangent map $T \phi$ of a smooth map $\phi: M \rightarrow N$.

A representation of a Lie algebroid $A$ is a vector bundle $E \rightarrow M$ together with a flat $A$-connection $\nabla$ (see, e.g, [4]). The usual operations $\oplus$ and $\otimes$ on vector bundles turn the space of representations $\operatorname{Rep}(A)$ into a semiring. Given a morphism of Lie algebroids $(\Phi, \phi)$, there is a pullback operation on representations $E \mapsto \phi^{!} E$, which gives a morphism of $\operatorname{rings} \phi^{!}: \operatorname{Rep}(B) \rightarrow \operatorname{Rep}(A)$.

For an orientable line bundle $L \in \operatorname{Rep}(A)$ the only characteristic class can be obtained as follows: for any nowhere vanishing section $\mu \in \Gamma(L)$,

$$
\nabla_{X} \mu=\left\langle\alpha_{\mu}, X\right\rangle \mu, \quad \forall X \in \mathfrak{X}(A) .
$$

The 1-form $\alpha_{\mu} \in \Omega^{1}(A)$ is $\mathrm{d}_{A}$-closed and it is called the characteristic cocycle of the representation $L$. Its cohomology class is independent of the choice of section $\mu$ and defines the characteristic class of the representation $L$ :

$$
\operatorname{char}(L):=\left[\alpha_{\mu}\right] \in H^{1}(A) .
$$

One checks easily that if $L, L_{1}, L_{2} \in \operatorname{Rep}(A)$, then:

$$
\operatorname{char}\left(L^{*}\right)=-\operatorname{char}(L), \quad \operatorname{char}\left(L_{1} \otimes L_{2}\right)=\operatorname{char}\left(L_{1}\right)+\operatorname{char}\left(L_{2}\right) .
$$

Also, if $(\Phi, \phi): A \rightarrow B$ is a morphism of Lie algebroids, and $L \in \operatorname{Rep}(B)$ then:

$$
\operatorname{char}\left(\phi^{!} L\right)=\Phi^{*} \operatorname{char}(L),
$$

where $\Phi^{*}: H^{\bullet}(B) \rightarrow H^{\bullet}(A)$ is the map induced by $\Phi$ at the level of cohomology. If $L$ is not orientable, then one defines its characteristic class to be the one half that of the representation $L \otimes L$, so the formulas above still hold, for non-orientable line bundles.

Every Lie algebroid $A \rightarrow M$ has a canonical representation in the line bundle $L_{A}=\wedge^{\mathrm{top}} A \otimes \wedge^{\mathrm{top}} T^{*} M$ :

$$
\nabla_{X}(\omega \otimes \mu)=£_{X} \omega \otimes \mu+\omega \otimes £_{\rho(X)} \mu .
$$

Then we set:

Definition 2.3. The modular cocycle of a Lie algebroid $A$ relative to a nowhere vanishing section $\omega \otimes \mu \in \Gamma\left(\wedge^{\text {top }} A \otimes \wedge^{\text {top }} T^{*} M\right)$ is the characteristic cocycle $\alpha_{\omega \otimes \mu}$ of the representation $L_{A}$. The modular class of $A$ is the characteristic class:

$$
\bmod (A):=\left[\alpha_{\omega \otimes \mu}\right] \in H^{1}(A) .
$$

Remark 2.4. Notice that, if $\nu=f \mu$ is another section of $L_{A}$, for a nonvanishing function $f \in C^{\infty}(M)$, then

$$
\alpha_{\nu}=\alpha_{\mu}-\mathrm{d}_{A} \ln f .
$$

Example 2.5. The modular class of a tangent bundle is trivial. 
Example 2.6. Let $(M, \pi)$ be a Poisson manifold. The first Poisson cohomology space $H_{\pi}^{1}(M)$, is the space of Poisson vector fields modulo the hamiltonian vector fields.

The Lie derivative of any volume form along hamiltonian vector fields leads to a unique vector field $X_{\mu} \in \mathfrak{X}(M)$ such that:

$$
£_{X_{f}} \mu=X_{\mu}(f) \mu .
$$

One calls $X_{\mu}$ the modular vector field of the Poisson manifold $(M, \pi)$ relative to $\mu$. The modular vector field $X_{\mu}$ is Poisson and, if $\nu=g \mu$ is another volume form, then:

$$
X_{g \mu}=X_{\mu}-\pi^{\sharp}(\mathrm{d} \ln |g|) .
$$

This lead to the definition of modular class of a Poisson manifold, which is due to Weinstein [12]:

The modular class of a Poisson manifold $(M, \pi)$ is the Poisson cohomology class

$$
\bmod (M):=\left[X_{\mu}\right] \in H_{\pi}^{1}(M) .
$$

Note that $\bmod (M)=0$ if and only if we can find a volume form $\mu$ invariant under all hamiltonian flows. Therefore the modular class is the obstruction to the existence of a volume form in $(M, \pi)$ invariant under all hamiltonian flows.

In fact, the modular class of the Poisson manifold $(M, \pi)$ and the modular class of the Lie algebroid $T^{*} M$ just differ by a multiplicative factor:

$$
\bmod \left(T^{*} M\right)=2 \bmod (M) .
$$

\section{The MODUlar ClASS OF A LIE ALGEBROID MORPHISM}

Let $\Phi: A \rightarrow B$ be a morphism of Lie algebroids covering a map $\phi: M \rightarrow N$. The induced morphism at the level of cohomology $\Phi^{*}: H^{\bullet}(B) \rightarrow H^{\bullet}(A)$, in general, does not map the modular classes to each other. Therefore one sets $([8])$ :

Definition 3.1. The modular class of a Lie algebroid morphism $\Phi: A \rightarrow B$ is the cohomology class defined by:

$$
\bmod (\Phi):=\bmod (A)-\Phi^{*} \bmod (B) \in H^{1}(A) .
$$

Proposition 3.2. Let $\Phi: A \rightarrow B$ and $\Psi: B \rightarrow C$ be Lie algebroid morphisms, then:

$$
\bmod (\Psi \circ \Phi)=\bmod (\Phi)+\Phi^{*} \bmod (\Psi)
$$

The basic properties for characteristic classes show that the modular class of a Lie algebroid morphism $(\Phi, \phi): A \rightarrow B$ can be seen as the characteristic class of a representation. Namely, one takes the canonical representations $L_{A} \in \operatorname{Rep}(A)$ and $L_{B} \in \operatorname{Rep}(B)$ and forms the representation $L^{\phi}:=L_{A} \otimes \phi^{!}\left(L_{B}\right)^{*}$. Then:

Proposition 3.3. Let $(\Phi, \phi): A \rightarrow B$ be a Lie algebroid morphism. Then:

$$
\bmod (\Phi)=\operatorname{char}\left(L^{\phi}\right)
$$

\section{The MOdular Class of A LIE ALGEBRoId COMORPHISM}

In this section we extend some of the results for Poisson maps in [2] to comorphisms between Lie algebroids. We begin with the definition of a Lie algebroid comorphism. Further details about comorphisms can be seen in $[10,1,11,13]$. 
Definition 4.1. Let $A \rightarrow M$ and $B \rightarrow N$ be two Lie algebroids. A comorphism between $A$ and $B$ covering $\phi: M \rightarrow N$ is a vector bundle map $\Phi: \phi ! B \rightarrow A$ from the pullback vector bundle $\phi^{!} B$ to $A$, such that the following two conditions hold:

$$
[\bar{\Phi} X, \bar{\Phi} Y]=\bar{\Phi}[X, Y]
$$

and

$$
\mathrm{d} \phi \circ \rho_{A}(\bar{\Phi} X)=\rho_{B}(X),
$$

for $X, Y \in \mathfrak{X}(B)$, where $\bar{\Phi}: \mathfrak{X}(B) \rightarrow \mathfrak{X}(A)$ is the natural map induced by $\Phi$.

Equivalently, we may say that $(\Phi, \phi)$ is a Lie algebroid comorphism if and only if $\Phi^{*}: A^{*} \rightarrow B^{*}$ is a Poisson map for the natural linear Poisson structures on the dual Lie algebroids.

Proposition 4.2. Let $\Phi: \phi^{!} B \rightarrow A$ be a Lie algebroid comorphism. The pullback vector bundle $\phi^{!} B \rightarrow M$ carries a natural Lie algebroid structure characterized by:

$$
\left[X^{!}, Y^{!}\right]=[X, Y]^{!}
$$

and

$$
\rho\left(X^{!}\right)=\rho_{A}\left(\bar{\Phi} X^{!}\right),
$$

for $X, Y \in \mathfrak{X}(B), X^{!}=X \circ \phi \in \Gamma\left(\phi^{!} B\right)$ and $Y^{!}=Y \circ \phi \in \Gamma\left(\phi^{!} B\right)$.

For this structure, the natural maps

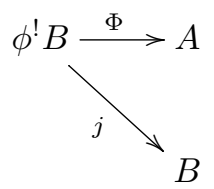

are Lie algebroid morphisms.

The modular class of a Lie algebroid comorphism is defined as follows:

Definition 4.3. Let $\Phi: \phi^{!} B \rightarrow A$ be a Lie algebroid comorphism between the Lie algebroids $A$ and $B$. The modular class of $\Phi$ is the cohomology class:

$$
\bmod (\Phi):=\Phi^{*} \bmod (A)-j^{*} \bmod (B) \in H^{1}\left(\phi^{!} B\right) .
$$

Example 4.4. A Poisson map $\phi: M \rightarrow N$ defines a comorphism between cotangent bundles: $\Phi: \phi^{!} T^{*} N \rightarrow T^{*} M$ such that $\Phi\left(\alpha^{!}\right)=(\mathrm{d} \phi)^{*} \alpha$, where $\alpha^{!}=\alpha \circ \phi \in$ $\mathfrak{X}\left(\phi^{!}\left(T^{*} N\right)\right)$, for all $\alpha \in \Omega^{1}(N)$. The modular class of the Poisson map $\phi$ was defined in [2] and we see that it is one half the modular class of the comorphism $\Phi$ induced by $\phi$.

Notice that the map $j^{*}: \Omega^{k}(B) \rightarrow \Omega^{k}\left(\phi^{!} B\right)$ is simply defined by

$$
j^{*}(\alpha)=\alpha \circ \phi, \quad \alpha \in \Omega^{k}(B) .
$$

Taking this into account we can give an explicit description of a representative of the modular class of a comorphism $\Phi$ :

Proposition 4.5. Let $\Phi: \phi^{!} B \rightarrow A$ be a Lie algebroid comorphism over $\phi: M \rightarrow N$ and fix non-vanishing sections $\mu \in \Gamma\left(L_{A}\right), \nu \in \Gamma\left(L_{B}\right)$. The modular class $\bmod (\Phi)$ is represented by:

$$
\alpha_{\mu, \nu}=\Phi^{*}\left(\alpha_{\mu}\right)-\alpha_{\nu} \circ \phi
$$

where $\alpha_{\mu}$ and $\alpha_{\nu}$ are the modular cocycle of $A$ and $B$ relative to $\mu$ and $\nu$, respectively.

We will refer to $\alpha_{\mu, \nu}$ as the modular cocycle of $\Phi$ relative to $\mu$ and $\nu$. 
Corollary 4.6. The class $\bmod (\Phi)$ is the obstruction to the existence of modular cocycles $\alpha \in \Omega^{1}(A)$ and $\beta \in \Omega^{1}(B)$, such that

$$
\Phi^{*} \alpha=\beta \circ \phi .
$$

Proof. The Lie algebroid morphism $\Phi$ has trivial modular class if its modular cocycles are exact in the Lie algebroid cohomology of $\phi^{!} B$, i.e., if for each $\mu \in \Gamma\left(L_{A}\right)$ and $\nu \in \Gamma\left(L_{B}\right)$,

$$
\alpha_{\mu, \nu}=\mathrm{d}_{\phi^{!} B} f=\Phi^{*}\left(\mathrm{~d}_{A} f\right), \quad \text { for some } f \in C^{\infty}(M)
$$

By definition $\alpha_{\mu, \nu}=\Phi^{*}\left(\alpha_{\mu}\right)-\alpha_{\nu} \circ \phi$, hence we have $\Phi^{*}\left(\alpha_{\mu}+\mathrm{d}_{A} f\right)=\alpha_{\nu} \circ \phi$, and taking into account equation (1), we conclude that $\alpha_{\mu}+\mathrm{d}_{A} f=\alpha_{e^{-f}}$ and $\Phi^{*} \alpha_{e^{-f} \mu}=X_{\nu}$.

Corollary 4.7. Let $\Phi: \phi^{!} B \rightarrow A$ be a comorphism between Lie algebroids. If there exists a Lie algebroid morphism $\widehat{\Phi}: A \rightarrow B$ making the diagram commutative

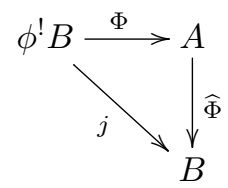

then

$$
\bmod \Phi=\Phi^{*} \bmod \widehat{\Phi}
$$

Proof. Since $j=\widehat{\Phi} \circ \Phi$ we have $j^{*}=\Phi^{*} \circ \widehat{\Phi}^{*}$ and

$$
\Phi^{*} \bmod \widehat{\Phi}=\Phi^{*}\left(\bmod A-\widehat{\Phi}^{*} B\right)=\Phi^{*} \bmod A-j^{*} \bmod B=\bmod \Phi .
$$

Proposition 4.8. Let $\Phi: A \rightarrow B$ be a comorphism between Lie algebroids. There is a natural representation of $\phi^{!} B$ on the line bundle $L^{\phi}:=L_{A} \otimes \phi^{!} L_{B}^{*}$, and we have:

$$
\bmod (\Phi)=\operatorname{char}\left(L^{\phi}\right)
$$

Proof. We define a representation of $\phi ! B$ on the line bundle $L_{A}$ by setting:

$$
\nabla_{X !}(\mu \otimes \nu):=\left[X^{!}, \mu\right]_{A} \otimes \nu+\mu \otimes £_{\rho_{A} \bar{\Phi} X} \nu
$$

and another representation on $\phi ! L_{B}$ by setting:

$$
\nabla_{X^{!}}\left(\mu^{!} \otimes \nu^{!}\right):=[\alpha, \mu]_{B}^{!} \otimes \nu^{!}+\mu^{!} \otimes\left(£_{\rho_{B}(X)} \nu\right)^{!},
$$

for $X \in \mathfrak{X}(B)$ and $\mu \otimes \nu \in \Gamma\left(L_{A}\right)$. The tensor product of the first representation with the dual of the second representation defines a representation of $\phi^{!} B$ on the line bundle

$$
L^{\phi}:=L_{A} \otimes \phi^{!} L_{B}^{*}
$$

Let us consider two Lie algebroids morphisms $\Phi: \phi^{!} B \rightarrow A$ and $\Psi: \psi^{!} C \rightarrow B$ over $\phi: M \rightarrow N$ and $\psi: N \rightarrow P$, respectively. The restriction $\widetilde{\Psi}=\Psi_{\mid(\psi \circ \phi) ! C}$ maps $(\psi \circ \phi)^{!} C$ to $\phi ! B$ and defines a map at the cohomology level:

$$
\widetilde{\Psi}^{*}: H^{\bullet}\left(\phi^{!} B\right) \rightarrow H^{\bullet}\left((\psi \circ \phi)^{!} C\right) .
$$

The function $\Psi \circ \Phi:(\psi \circ \phi)^{!} C \rightarrow A$ defined by:

$$
\Psi \circ \Phi\left(X_{\psi \circ \phi(m)}\right)=\Phi\left(\widetilde{\Psi}\left(X_{\psi \circ \phi(m)}\right)\right), \quad(\forall m \in M),
$$

is a Lie algebroid comorphism. 
We also have the natural Lie algebroid morphism $\tilde{j}:(\psi \circ \phi)^{!} C=\phi^{!} \psi^{!} C \rightarrow \psi^{!} C$ that defines a map at the cohomology level

$$
\tilde{j}^{*}: H^{\bullet}\left(\psi^{!} C\right) \rightarrow H^{\bullet}\left((\psi \circ \phi)^{!} C\right), \alpha \mapsto \alpha \circ \phi .
$$

Proposition 4.9. Let $\Phi: \phi^{!} B \rightarrow A$ and $\Psi: \psi^{!} C \rightarrow B$ be Lie algebroid comorphisms. Then:

$$
\bmod (\Psi \circ \Phi)=\widetilde{\Psi}^{*} \bmod (\Phi)+\tilde{j}^{*} \bmod (\Psi) .
$$

Proof. The following diagram commutes:

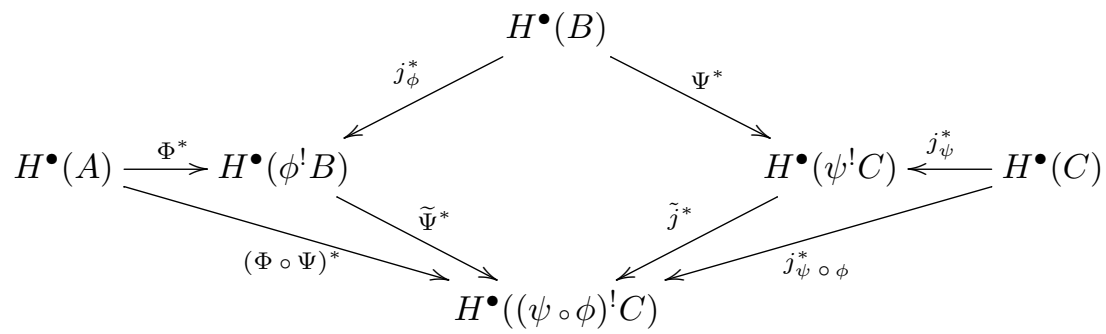

Hence, we find:

$$
\begin{aligned}
\bmod (\Phi \circ \Psi) & =(\Phi \circ \Psi)^{*} \bmod (A)-j_{\psi \circ \phi}^{*} \bmod (C) \\
& =\widetilde{\Psi}^{*} \circ \Phi^{*} \bmod (A)-\tilde{j}^{*} \circ j_{\psi}^{*} \bmod (C) \\
& =\widetilde{\Psi}^{*} \circ \Phi^{*} \bmod (A)-\widetilde{\Psi}^{*} \circ j_{\phi}^{*} \bmod (B)+\widetilde{\Psi}^{*} \circ j_{\phi}^{*} \bmod (B)-\tilde{j}^{*} \circ j_{\psi}^{*} \bmod (C) \\
& =\widetilde{\Psi}^{*}\left(\Phi^{*} \bmod (A)-j_{\phi}^{*} \bmod (B)\right)+\tilde{j}^{*}\left(\Psi^{*} \bmod (B)-j_{\psi}^{*} \bmod (C)\right) \\
& =\widetilde{\Psi}^{*} \bmod (\Phi)+\tilde{j}^{*} \bmod (\Psi) .
\end{aligned}
$$

\section{Generalization to Dirac structures}

The modular class of a Lie algebroid morphism and the modular class of a Lie algebroid comorphism fit together into the notion of modular class of a skew algebroid relation, given by Grabowski in [5]. As a particular case we have the modular class of a Dirac map but very few was said about this particular case. The study of these structures will be exposed in a future work.

\section{REFERENCES}

[1] A. Cattaneo, B. Dherin and A. Weinstein, Integration of Lie algebroid comorphisms. Eprint arXiv: arXiv: 1210.4443 .

[2] R. Caseiro and R.L. Fernandes, Modular class of Poisson maps. Eprint arXiv: arXiv:1103.4305.

[3] S. Evens, J.-H. Lu and A. Weinstein, Transverse measures, the modular class and a cohomology pairing for Lie algebroids. Quart. J. Math. Oxford (2) 50 (1999), 417-436.

[4] R.L. Fernandes, Lie algebroids, holonomy and characteristic classes. Adv. Math. 170 (2002), no. $1,119-179$.

[5] J. Grabowski, Modular classes of skew algebroid relations. Transform. Groups 17 (2012), no. 4, 989-1010.

[6] J. Grabowski, G. Marmo and P. Michor, Homology and modular classes of Lie algebroids. Ann. Inst. Fourier 56 (2006), 69-83.

[7] Y. Kosmann-Schwarzbach and A. Weinstein, Relative modular classes of Lie algebroids. C. R. Math. Acad. Sci. Paris 341 (2005), no. 8, 509-514.

[8] Y. Kosmann-Schwarzbach, C. Laurent-Gengoux and A. Weinstein, Modular classes of Lie algebroid morphisms. Transform. Groups 13 (2008), no. 3-4, 727-755.

[9] J.-L. Koszul, Crochet de Schouten-Nijenhuis et cohomologie.Asterisque Numero Hors Serie (1985), 257-271.

[10] K. Mackenzie, General theory of Lie goupoids and Lie algebroids, London Mathematical Society Lecture Notes Series 213, Cambridge University Press (2005). 
[11] P. Stachura, $C^{*}$-algebra of a differential groupoid, Banach Center Publ. 51 (1998), no. 1, 263-281.

[12] A. Weinstein, The modular automorphism group of a Poisson manifold. J. Geom. Phys. 23 (1997), no. 3-4, 379-394.

[13] S. Zakrzewski, Quantum and classical pseudogroups. Part II. Differential and Symplectic Pseudogroups, Comm. Math. Phys. 134 (1990), no. 2, 371-395.

CMuC, Departamento de Matemática, Faculdade de Ciências e Tecnologia, Universidade de Coimbra, Apartado 3008, 3001-454 Coimbra, Portugal

E-mail address: raquel@mat.uc.pt 